Nouvelles perspectives en sciences sociales

\title{
Analyses qualitatives et quantitatives : deux visions, une même science
}

\section{Simon Laflamme}

Volume 3, numéro 1, septembre 2007

URI : https://id.erudit.org/iderudit/602467ar

DOI : https://doi.org/10.7202/602467ar

Aller au sommaire du numéro

\section{Éditeur(s)}

Prise de parole

\section{ISSN}

1712-8307 (imprimé)

1918-7475 (numérique)

Découvrir la revue

\section{Citer cet article}

Laflamme, S. (2007). Analyses qualitatives et quantitatives : deux visions, une même science. Nouvelles perspectives en sciences sociales, 3(1), 141-149. https://doi.org/10.7202/602467ar
Résumé de l'article

Cet article suggère que, malgré bon nombre de plaidoiries qui affirment que les méthodes qualitatives et quantitatives sont antinomiques, ces deux types de méthode ont beaucoup en partage et ils sont tous les deux soumis aux mêmes exigences de scientificité. Non seulement jouent-ils des rôles complémentaires dans les sciences humaines, mais, en plus, 1) leurs analyses doivent toujours avoir quelque représentativité, 2) leurs résultats doivent pouvoir caractériser un ensemble étudié, 3) les caractérisations doivent être modulées par les variations individuelles, 4) leurs analyses doivent établir des liens entre les objets/sujets étudiés, et 5) ces analyses sont soumises à la tension qui s'impose entre l'obligation de regrouper les données et celle d'en respecter les particularités. 


\title{
Analyses qualitatives et quantitatives : deux visions, une même science
}

\author{
SiMON LAFLAMME \\ Université Laurentienne
}

Le vocabulaire de l'analyse de données textuelles n'est pas le même que celui de l'analyse de données quantitatives. Pourtant toutes deux se soumettent à des principes très semblables. C'est l'opinion que nous proposons dans le texte qui suit. Car il nous semble que les positions idéologiques antiqualitativistes ou antiquantitativistes nuisent aussi bien au développement des sciences humaines dans leur ensemble qu'à l'intelligence du travail qui est effectué quand on recourt aux diverses méthodes qu'offrent ces sciences.

Il faut dire que ces deux modes analytiques se sont souvent constitués en opposition l'un par rapport à l'autre. Bon nombre d'adeptes de l'approche quantitative ont longtemps exprimé du mépris pour l'analyse qualitative, la percevant comme une forme inférieure. Bon nombre de protagonistes de l'approche qualitative ont eux-mêmes dédaigné l'analyse statistique, lui reprochant de ne pas pouvoir tenir compte de divers phénomènes, voire de déformer la réalité, notamment humaine. Dans leurs manifestations les plus naïves, la critique quantitativiste réduit la recherche qualitative à une énonciation subjective et associe science à mathématisation, la critique qualitativiste, elle, assimile positivisme - entendant par là vaine prétention à l'objectivité - à statistique et nie le bien-fondé de la quantification de la vie humaine et sociale. La critique quantitativiste ignore alors que toute analyse du réel, quelle qu'elle soit, est sociale et historique et que toute modélisation, 
mathématique ou non, est désubjectivation dès lors qu'elle se soumet à des impératifs de logique, d'adéquation entre le discours sur l'objet et l'objet lui-même et de communication des résultats dans un champ polémique. La critique qualitativiste oublie que s'il est vrai que toute connaissance est sociale et historique, cela ne signifie pas qu'elle soit subjective et que la vision subjectiviste des sciences, même humaines, est aussi illusoire que la vision objectiviste : si la science construit l'univers qu'elle observe, elle ne le fait pas en tant que cet univers puisse être la simple expression d'un sujet. La critique qualitativiste oublie aussi de remarquer que ses modèles d'analyse, si peu mathématiques soientils, n'en sont pas moins des constructions du monde au même titre que le sont les modèles statistiques. Il n'y a pas d'univers observable brut. Rechercher, chez un acteur social, ses motifs, c'est le modéliser en tant qu'objet agissant en fonction de motifs. Affirmer que l'acteur est conscient, rationnel, intentionnel, stratégique, c'est le modéliser en tant que tel. L'analyse qualitative n'est pas pure correspondance entre l'interprétation et l'objet, pas plus que ne l'est l'analyse statistique. Toutes deux modélisent le monde observable ; aucune n'est pour autant ni pure subjectivité ni pure objectivité.

Toute vision hiérarchique ou exclusive de ces deux approches est litigieuse, à moins qu'elle ne s'inscrive dans le cadre circonstanciel d'une problématique de recherche particulière. L'analyse qualitative, dans le champ des sciences humaines, complète l'analyse quantitative, et vice versa. Toutes deux permettent d'accéder à des informations spécifiques qui enrichissent les connaissances. Souvent elles permettent de vérifier diversement une hypothèse comparable. Souvent elles ouvrent à la recherche des univers dissemblables. Dans certains cas, l'une devient une manière de vérifier ce qui a été découvert avec l'autre. Parfois, par exemple, l'analyse statistique aura permis d'établir des corrélations sur de grands échantillons et l'analyse qualitative, elle, permettra d'observer ce qu'il en est de manière quasi expérimentale au niveau de cas particuliers ; parfois, les observations d'une analyse qualitative demanderont à être extrapolées à des populations, ce que rendra possible l'analyse statistique. Ce sont là des évidences que seul l'aveuglement de quelques idéologues empêche de reconnaître. Mais ces évidences ne sont pas telles qu'elles admettent la comparabilité des approches qualitative et quantitative au plan scientifique. Il suffit de prendre l'index d'un manuel d'analyse qualitative et celui d'un manuel 
d'analyse quantitative pour remarquer leur dissimilitude, même pour ce qui est du lexique le plus élémentaire. L'index d'un manuel de statistique comporte obligatoirement le mot variable ${ }^{1}$; celui d'un manuel d'analyse qualitative, non ${ }^{2}$, à moins que ce ne soit pour renvoyer à une mention furtive $^{3}$ ou à une dénonciation du vocabulaire de l'autre $e^{4}$ ou pour décrire des techniques qui côtoient la statistique, comme c'est le cas pour l'analyse de contenu ${ }^{5}$. Pourtant, les deux approches reposent sur des principes similaires qu'on peut regrouper dans cinq champs énonciatifs.

\section{Les données doivent avoir quelque représentativité}

La plupart des analyses, quantitatives ou qualitatives, portent sur un échantillon. Un échantillon, par essence, doit représenter quelque population et rendre possible quelque inférence. En fonction des particularités de la recherche et des contraintes de tous ordres qui s'imposent, le chercheur peut recourir à diverses techniques

David C. Howell, Méthodes statistiques en sciences bumaines, De Boeck Université, [1997] 1998.

Norman K. Denzin et Yvonna S. Lincoln (dir.), Collecting and interpreting qualitative materials, Thousand Oaks, London, New Delhi, Sage Publications, 2003. Norman K. Denzin et Yvonna S. Lincoln (dir.), Handbook of qualitative research, Thousand Oaks, London, New Delhi, Sage Publications, 1994 et $2^{c}$ édition, 2000. Uwe Flick, $A n$ introduction to qualitative research, $3^{c}$ édition, London, Thousand Oaks, New Delhi, Sage Publications, [1998] 2006. Paul ten Have, Understanding qualitative research and ethnomethodology, Jondon, Thousand Oaks, New Delhi, Sage Publications, 2004. Joseph A. Maxwell, Qualitative research design: An interactive approach, 2 édition, Thousand Oaks, London, New Delhi, Sage Publications, Applied social research methods series, vol. 41, 2005.

Jean Poupart, Jean-Pierre Deslauniers, Lionel-Henri Groulx, Anne I-aperrière, Robert Mayer et Alvaro P. Pires, La recherche qualitative. Enjeux épistémologiques et méthodologiques, Montréal, Paris, Casablanca, Gaëtan Morin, 1997.

4 David Silverman, Interpreting qualitative data: Methods for analysing talk, text, and interaction, London, Thousand Oaks, New Dehli, Sage Publications, 1993, 2006.

Bruce L. Berg, Qualitative research metbods for social sciences, $2 *$ édition, Boston, Allyn and Bacon, [1989] 1995. 
échantillonales, qu'elles soient probabilistes ${ }^{6}$ ou non ${ }^{7}$. Ces techniques sont à la disposition des traitements aussi bien quantitatifs que qualitatifs. Le recours à une technique non probabiliste n'invalide pas automatiquement les résultats qui sont obtenus; il invite à la prudence toute extrapolation qu'on voudrait en faire. Certains qualitativistes refusent par principe de parler d'échantillon pour désigner les individus (au sens large) sur lesquels porte leur analyse. Dans la mesure où ces analyses n'ont pour fin que de dépeindre les individus examinés - qui deviennent ainsi population -, le refus peut se comprendre ; mais dès lors que ces individus deviennent quelque indication de quelque groupe plus étendu, ils deviennent de facto échantillon. Pour estimer la portée des résultats qu'elle obtient, l'analyse quantitative recourt à des techniques inférentielles - paramétriques ou non paramétriques - dont la validité, si mathématique soit-elle, dépend directement des techniques échantillonales - le plus beau test d'inférence ne vaut rien si l'on ne peut compter sur la représentativité de l'échantillon. L'analyse qualitative, elle, n'emploie pas de test d'inférence - à moins qu'elle ne quantifie ses observations. Mais elle est bien armée pour vérifier si un échantillon a quelque représentativité ; elle vérifie l'effet de saturation. Elle est ainsi à même de déclarer que les résultats auxquels elle parvient correspondent à la population puisqu'elle a noté qu'en ajoutant d'autres individus à l'ensemble de ceux qui font partie de l'analyse, elle n'accroît pas le nombre des informations par rapport à celles qu'elle a déjà obtenues. La dissimilitude entre l'analyse qualitative et l'analyse statistique n'est donc pas que, pour l'une, la question de l'échantillon est importante alors qu'elle ne le serait pas pour l'autre ; elle est bien simplement dans la manière de prendre en considération cette préoccupation scientifique. L'une et l'autre sont toujours tenues de déterminer la portée des résultats obtenus ; l'une et l'autre doivent toujours extrapoler avec prudence les résultats qui ne sont pas obtenus sur la population elle-même.

"Échantillons aléatoire simple, aréolaire, en grappes, stratifié ; échantillon par répartition aléatoire des individus.

Par quota, accidentels, de volontaires, systématiques, par choix raisonné, boule de neige. 


\section{Les résultats doivent pouvoir caractériser l'ensemble étudié}

En analyse qualitative ou quantitative, une description doit pouvoir caractériser l'objet étudié, qu'il soit pluriel ou singulier. En analyse quantitative, on peut recourir à des mesures de tendance centrale : le mode, la médiane ou la moyenne. On emploie le mode pour les données nominales et on l'exprime tout simplement en fréquences, relatives ou absolues. On fait appel à la médiane pour les variables ordinales, où est indiqué le rang. On utilise la moyenne pour les variables cardinales. Les mesures de tendance centrale pour les variables des niveaux statistiquement inférieurs valent pour les variables des niveaux supérieurs. L'analyse qualitative, notamment celle qui se penche sur des données textuelles, ne se sert pas de mesures de tendance centrale à proprement parler, elle crée des catégories, lesquelles sont des regroupements d'informations, de propos ou d'énoncés par exemple. Appartiendront à une même classe des énoncés récurrents ou encore des terminologies ou des propositions sémantiquement semblables. La catégorisation est ainsi une façon particulière de réunir les informations, de faire état des tendances dans un corpus particulier. On notera, au passage, que l'analyse de données textuelles peut emprunter des outils statistiques et, partant, mesurer des tendances centrales. La dissimilitude entre les deux modes analytiques se révèle donc dans la manière de réunir les informations et d'accéder à ce qui est central, et non dans le fait lui-même de devoir les regrouper et d'en dégager l'essentiel. Là, par ailleurs, où ces deux modes se distinguent le plus l'un de l'autre, c'est dans les facultés herméneutiques de l'analyse qualitative. Les données dont elle dispose et sa manière de réunir de l'information lui permettent de découvrir du sens dans les corpus étudiés bien au-delà de ce à quoi pourrait accéder l'analyse quantitative. Les outils quantitatifs peuvent servir une analyse de données textuelles ; ils peuvent le faire notamment par l'intermédiaire de logiciels comme Tropes, Alceste, N-Vivo, Lexico, $S P A D$ en procédant à des traitements lexicaux, syntaxiques ou morphosyntaxiques. Mais l'analyse quantitative, en elle-même, n'a qu'exceptionnellement des visées herméneutiques alors que l'analyse qualitative recueille normalement ses données dans le but de découvrir une manière de penser; c'est pourquoi ses données doivent souvent être ce qu'exprime un répondant selon sa propre formulation. 


\section{Les caractérisations de groupe doivent être modulées par les variations entre les individus}

Les mesures de tendance centrale, en statistique, s'accompagnent de mesures de dispersion. Ces mesures de dispersion permettent d'informer sur la distribution à partir de laquelle a été obtenue la tendance centrale. Une même moyenne, par exemple, peut caractériser deux ensembles nettement différents. L'écart type, entre autres, indiquera dans quelle mesure les scores des individus sont ou non concentrés autour de la moyenne. En inférence, ces mesures qui permettent de prendre en considération la différence entre les individus dans les groupes (voire entre les groupes) peuvent empêcher l'inférence des variations entre les groupes. Une mesure de dispersion très élevée, qui signale une grande différence entre les individus, oblige à nuancer le jugement qui est porté sur le groupe quand on tient compte de la seule tendance centrale. Il n'en va pas autrement en analyse qualitative, les variations intra-individuelles ou inter-individuelles complexifient le travail de catégorisation. Plus les ensembles sont hétérogènes, plus il est difficile de créer des catégories qui les caractérisent.

\section{Il faut établir des liens entre les objets observés}

Les principes de la causalité sont les mêmes pour l'analyse qualitative que pour l'analyse quantitative. Il faut rendre compte dans les deux cas des variations, il faut expliquer ce qui différencie ou ce qui rend semblable, ou encore il faut expliquer un événement. Les deux types d'analyse peuvent, par exemple, établir qu'il y a corrélation entre l'opinion et le sexe, que les hommes ne pensent pas comme les femmes, que la variable sexe, donc, fait varier la variable opinion. Dans les deux cas, une telle conclusion n'est autorisée que s'il est bien montré qu'hommes et femmes ne pensent pas semblablement, ce à quoi ne permet pas de conclure, par exemple, une analyse qui ne porterait que sur les hommes ou que sur les femmes, à moins que cette analyse soit arrimée à une autre où l'autre sexe a déjà été examiné. Une analyse sur un seul objet ne permet pas de tirer des conclusions sur la spécificité de cet objet lui-même. Seule une comparaison rend cette distinction possible. Cette comparaison peut être assumée ou non dans le devis de recherche, mais elle est toujours requise, et, pour cette raison, il est 
préférable que le devis la prévoie. Une comparaison est la prise en considération d'une variable, celle qui comprend les ensembles comparés (les sexes, les régions, les groupes d'âge...). Cette comparaison suppose en outre l'examen d'au moins une autre variable, celle à partir de laquelle la comparaison sera effectuée (l'opinion, par exemple). $\mathrm{Ne}$ pas employer le vocabulaire, ce n'est pas ne pas faire la chose. Établir un lien entre deux variables, ni en analyse qualitative, ni en analyse quantitative, c'est démontrer que la variable indépendante est cause des variations de la variable dépendante. Quel que soit le mode d'analyse, il importe toujours de vérifier les corrélations obtenues soit en se donnant les moyens d'observer la mécanique du lien, soit en recourant à des variables intermédiaires.

\section{Il ne faut pas tant regrouper qu'on perde de l'information, il ne faut pas tant coller à l'information qu'on ne puisse regrouper}

L'analyse statistique comme l'analyse qualitative doivent décrire et expliquer. La description ne peut pas consister en un simple rapport de l'ensemble des données qui ont été recueillies; il n'y aurait pas, de cette façon, d'analyse. L'information recueillie demande à être regroupée en des ensembles, à se révéler dans des tendances, sinon elle est informe, et donc inutile. Le spécialiste des sciences sociales n'est pas un rapporteur, il est un analyste. À ce titre, il doit donner une forme à la matière qui se donne à lui, ou qu'il se donne lui-même, et il doit pouvoir en expliquer les variations. Mais tout scientifique est toujours aux prises avec un indépassable dilemme, lequel est bien connu : il lui faut regrouper suffisamment l'information pour découvrir du sens; il ne lui faut pas à ce point regrouper qu'il perde de vue l'information. À créer des ensembles trop grands, on finit par nier les éléments constitutifs; à trop coller aux éléments, on ne parvient pas à saisir ce qui les rend semblables ou non. Ce paradoxe vaut pour toute analyse, qu'elle soit qualitative ou quantitative. Le regroupement est en soi l'amorce d'une explication parce qu'il est introduction de sens, mais cette explication prendra elle-même une forme supérieure à partir du moment où les regroupements seront mis en relation les uns avec les autres. La science porte sur des catégories, mais elle examine des liens. Dans la mesure des 
contraintes empiriques d'une recherche, ces liens ne doivent pas s'établir entre des ensembles à ce point abstraits qu'ils n'ont plus de référent.

\section{Conclusion}

L'analyse qualitative n'est pas l'analyse quantitative. Toutes deux sont essentielles au développement des sciences humaines. Certains discours, par contre, donnent à croire que l'une et l'autre sont radicalement dissemblables au plan scientifique. Nous avons voulu, dans ce texte, rappeler qu'elles répondent toutes deux à des logiques semblables, celles de la rigueur scientifique, malgré la spécificité de leurs objets. L'une et l'autre doivent constamment se poser la question de la représentativité des résultats obtenus, celle de la tendance des informations qui appartiennent à l'ensemble étudié, celle du rapport entre cette tendance et les spécificités des individus à partir desquels elle est dégagée, celle de la cause des variations observée et celle de l'abstraction. La vérité n'est pas en soi quantification. La quantification peut conduire à la vérité. Mais elle n'est pas la seule à pouvoir le faire ; l'observation rigoureuse de données textuelles en est tout aussi capable. Le vocabulaire de ces deux modes analytiques peut donner l'impression que l'analyse qualitative et l'analyse quantitative diffèrent dans leur scientificité même bien que, fondamentalement, elles sont soumises aux mêmes impératifs.

\section{Bibliographie}

Berg, Bruce L., Qualitative research methods for social sciences, $2^{c}$ édition, Boston, Allyn and Bacon, [1989| 1995.

Denzin, Norman K. et Yvonna S. I.incoln (dir.), Collecting and interpreting qualitative materials, Thousand Oaks, London, New Delhi, Sage Publications, 2003.

Denzin, Norman K. et Yvonna S. Lincoln (dir.), Handbonk of qualitative research, Thousand Oaks, London, New Delhi, Sage Publications, 1994 et $2^{c}$ édition, 2000

Flick, Uwe, $A n$ introduction to qualitative research, 3 édition, London, Thousand Oaks, New Delhi, Sage Publications, [1998] 2006.

Have, Paul ten, Understanding qualitative research and ethnomethodology, London, Thousand Oaks, New Delhi, Sage Publications, 2004.

Howell, David C., Méthodes statistiques en sciences bumaines, De Boeck Université, [1997] 1998. 
Maxwell, Joseph A., Qualitative research design: A interactive approach, $2^{*}$ édition, Thousand Oaks, London, New Delhi, Sage Publications, Applied social research methods series, vol. 41, 2005.

Poupart, Jean, Jean-Pierre Deslauniers, Lionel-Henri Groulx, Anne Laperrière, Robert Mayer et Alvaro P. Pires, La recberche qualitative. Enjeux epistémologiques et méthodologiques, Montréal, Paris, Casablanca, Gaëtan Morin, 1997.

Silverman, David, Interpreting qualitative data: Methods for analysing talk, text, and interaction, London, Thousand Oaks, New Dehli, Sage Publications, 1993, 2006. 